\title{
The Cluster Expansion in Statistical Mechanics*
}

\author{
David Brydges *ᄎ and Paul Federbush \\ Department of Mathematics, University of Michigan, Ann Arbor, Michigan 48109, USA
}

\begin{abstract}
The Glimm-Jaffe-Spencer cluster expansion from constructive quantum field theory is adapted to treat quantum statistical mechanical systems of particles interacting by finite range potentials. The Hamiltonian $H_{0}+V$ need be stable in the extended sense that $H_{0}+4 V+B N \geqq 0$ for some $B$. In this situation, with a mild technical condition on the potentials, the cluster expansion converges and the infinite volume limit of the correlation functions exists, at low enough density. These infinite volume correlation functions cluster exponentially. We define a class of interacting boson and fermion particle theories with a matter-like potential, $1 / r$ suitably truncated at large distance. This system would collapse in the absence of the exclusion principle-the potential is unstable--but the Hamiltonian is stable. This provides an example of a system for which our method proves existence of the infinite volume limit, that is not covered by the classic work of Ginibre, which requires stable potentials.

One key ingredient is a type of Holder inequality for the expectation values of spatially smeared Euclidean densities, a special interpolation theorem. We also obtain a result on the absolute value of the fermion measure, it equals the boson measure.
\end{abstract}

\section{Introduction}

In the quantum statistical mechanical theory of matter (positive charged particles and negative charged identical fermions interacting with a $1 / r$ potential) the most basic result is the stability, first proved by Dyson and Lenard in [2]. One of the authors presented a new proof in [3], and recently another proof was given by Lieb and Thirring in [8]. The second basic result was the proof of the existence of the thermodynamic functions in the infinite volume, by Lieb and Lebowitz in [7]. A natural next problem is the existence of the infinite volume correlation functions, for some range of parameters - an open question.

* This work was supported in part by NSF Grant MPS 75-10751

** Michigan Junior Fellow 
Towards this end, the Glimm-Jaffe-Spencer cluster expansion (see [6]) was adapted to treat the problem of matter with the $1 / r$ interaction modified to $1 / r$ $\left(e^{-\alpha r}-e^{-\beta r}\right)$ in [4]. In this situation (with suitable values of $\alpha, \beta$, etc.) the cluster expansion was shown to converge, yielding the existence of the infinite volume correlation functions. However, the classical methods of Ginibre (see [5]) already applied to this case, so this was not a new result.

In a later paper we will show that for a matter-like system with $1 / r$ replaced by $e^{- \text {ar }} / r$ the infinite volume limit of the correlation functions exists, (for some range of parameters). This will be a straight forward extension of the present paper and [4]. At present we consider a matter-like system with $1 / \mathrm{r}$ modified to

$v(x-y)=\int d^{3} z f(x-z)|x-y|^{-1} f(y-z)$

with $f$ a non-negative function in $C_{0}^{2}$. For this system, the Hamiltonian is stable, $H+B N \geqq 0$

for some $B$, but the potential is not. The system would collapse in the absence of the exclusion principle. We derive the existence of the infinite volume correlation functions for this system (in some range of parameters).

In fact our treatment is much more general than just of the aforementioned matter-like systems. We consider systems of boson and fermion particles interacting via two-body potentials with $H=H_{0}+V$. In this paper we assume

a) The potentials are finite range.

b) $H_{0}+4 V$ is stable, i.e. there is $B^{\prime}$ such that

$$
H_{0}+4 V+B^{\prime} N \geqq 0 \text {. }
$$

c) The potentials are in $L_{3 / 2}$.

Our main result will be that for such a system at any temperature, if $\mu$, the chemical potential, is large enough negative the infinite volume limit of the correlation functions exists. We do not detail the need for condition c) in this paper, a technical condition to justify some of the basic manipulations.

Section 2 presents the cluster expansion we use. Familiarity with [6] is assumed. Section 3 contains a statement of our basic results. The key steps in the proof of convergence are given in Section 4. Appendix A contains a proof that the absolute value of the fermion measure equals the boson measure. Appendix $B$ discusses the stability of our matter-like system. Appendices D and E contain technical estimates important to the convergence argument.

The key to the efficiency of the present paper is the interpolation estimate in Appendix C. It gives a very useful analog of the Holder inequality for systems with fermions, where the natural setting is function spaces over signed measures, rather than measures as with pure boson theories. We believe it goes a long way in bridging the gap between techniques available for fermion theories and techniques for boson theories.

The cluster expansion as developed here is purely a geometric analysis of the paths that realize the traces in path space. The total path space integral is split into subsets in which paths avoid certain regions and must hit other regions. The use of barrier potentials as in [4] is bypassed, this is a matter of choice. 
In addition to the generalization to infinite range potentials mentioned above, that will be the subject of a further paper, it is trivial to include finite range manybody forces in the present treatment.

\section{Notation and the Cluster Expansion}

We consider $l$ species of particles described by fields $\phi_{1}(x), \ldots, \phi_{l}(x)$ obeying either fermion or boson statistics. Let

$$
\begin{aligned}
H_{00} & =\sum_{i=1}^{l}\left(1 / 2 m_{i}\right) \int d x\left(\nabla \bar{\phi}_{i}\right)(x)\left(\nabla \phi_{i}\right)(x) \\
H_{0} & =H_{00}-\sum_{i=1}^{l} \mu_{i} \int d x \bar{\phi}_{i}(x) \phi_{i}(x) \\
N_{i} & =\int d x \bar{\phi}_{i}(x) \phi_{i}(x) ; \quad N=\sum_{i=1}^{l} N_{i} \\
H & =H_{0}+V
\end{aligned}
$$

$V$ is constructed from potentials with finite range. To partially fix the length scale assume the range is less than $2 / 10$. We will assume that $V$ is sufficiently regular that Friedrichs extensions $H^{\boldsymbol{A}}$ may be defined by extending $H$ off $N$-particle wave functions with compact support in an open bounded region $(A)^{N} \subset \mathbb{R}^{3 N}$; and furthermore that $\exp \left(-\beta H^{\Lambda}\right)$ admits a path space representation (FeynmanKac formula) on $N$-particle subspaces.

$\mathbb{R}^{3}$ is filled with closed unit cubes $\left\{\Delta_{i}\right\}$ with disjoint interiors. $\Lambda$ (the large box one works in) is the interior of a finite union of such cubes. The cluster expansion is applied to quantities of the form

$$
\langle A\rangle_{A}=\operatorname{Tr}_{\Lambda}\left(\operatorname{Te}^{-\int_{0}^{\beta} H^{\Lambda}(\tau) d \tau} A\right) / \operatorname{Tr}_{A}\left(e^{-\beta H^{\Lambda}}\right)
$$

where $\operatorname{Tr}_{A}$ is the trace on the Fock space built on $L^{2}(A) . T$ is the time-ordering operator. $A$ has the form

$A=a_{1}\left(t_{1}\right) \ldots a_{s}\left(t_{s}\right)$

where

$$
a_{i}\left(t_{i}\right)=\sum_{j=1}^{l} \int d x f_{i j}(x) \bar{\phi}_{j}(x) \phi_{j}(x) .
$$

Thus the $t_{i}$ is dummy, it serves to define the order of the operators in (2.5). For a given $i$ each $f_{i j}$ is supported in a single cube $\Delta$ for all $j=1, \ldots, l$. Each $f_{i j}$ is real, measurable, and $0 \leqq f_{i j} \leqq 1$, With these conditions our estimates may be taken to depend on the operator $A$ only through the number of factors, $s$.

The expression (2.5) can be represented as a path integral using a signed measure. Thus

$$
\operatorname{Tr}_{A}\left(\operatorname{Te}^{-\int_{0}^{\beta} H^{A}(\tau) d \tau} A\right)=\int_{A} d \mu e^{-\int_{0}^{\beta} V(\tau) d \tau} a_{1}\left(t_{1}\right) \ldots a_{s}\left(t_{s}\right)
$$


$d \mu$ may be described in the following laborious way: $d \mu=\prod_{j=1}^{l} d \mu^{(j)}$ where $d \mu^{(j)}$ is associated with the ${ }^{j}$ th species. Then $d \mu^{(j)}=\bigoplus_{N=0}^{\infty} d \mu_{N}^{(j)}$ where the $N$ particle measure $d \mu_{N}^{(j)}$ is

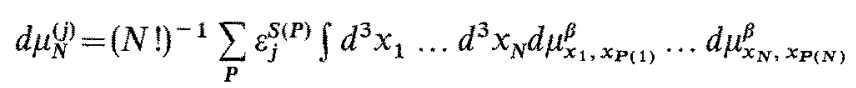

$d \mu_{x, y}^{B}$ is the measure on the space of paths $t \rightarrow x_{t} \in \mathbb{R}^{3}$ (starting at $x$ at $t=0$ and ending at $y$ at $t=\beta)$ associated with the semigroup $\exp -t\left(-\mu_{j}-\Delta\right) .\left(d \mu_{0}^{(j)} \equiv 1\right)$. $P$ is a permutation of $\{1,2, \ldots, N\} \cdot \varepsilon_{j}=\left(\begin{array}{c}1 \\ -1\end{array}\right)$ if species $j$ is $\left(\begin{array}{c}\text { boson } \\ \text { fermion }\end{array}\right) \cdot S=\left(\begin{array}{l}0 \\ 1\end{array}\right)$ if $P$ is $\left(\begin{array}{l}\text { even } \\ \text { odd }\end{array}\right)$. The integral over $x_{1}, \ldots, x_{N}$ takes the trace. The $A$ on the integral sign in (2.8) means that the integration is over the subset of path space such that the paths of each particle do not hit $\Lambda^{c}$ in the time interval $[0, \beta] . V(\tau), a_{i}\left(t_{i}\right)$ in (2.8) stand for the obvious functions corresponding to the operators $V, a_{i}$ evaluated at the positions of the paths for each particle at times, $\tau, t_{i \cdot}$. On an $n$-particle subspace the $n$ paths describing the particles give a mapping $t \rightarrow R^{3 n}$ which we call an $n$-path.

Our description of the cluster expansion imposes the following notation. $\left\{S_{\alpha}\right\}$ is the set of all faces of cubes $\left\{\Delta_{i}\right\} . E_{\alpha}$ is the characteristic function of the subset of path space consisting of all $n$-paths such that no particle hits the "barrier" $\eta_{\alpha}=\left\{x \in \mathbb{R}^{3}:\right.$ dist $\left.\left(x, S_{\alpha}\right) \leqq \frac{1}{10}\right\}$ in the time interval $[0, \beta]$. Note that the width of the barrier is greater than the range of $V . X \subset A$ is a union of cubes $\Delta_{i} \cdot\left\{\Delta_{j}: j \in J\right\}$ is a distinguished set of cubes.

The cluster expansion is developed by inserting inside the $d \mu$ integral in (2.8) the identity $1=\prod_{\alpha}\left(E_{\alpha}+H_{\alpha}\right)$ where $H_{\alpha}=1-E_{\alpha}$ and $\alpha$ runs over faces $S_{\alpha}$ in $A$, then expanding the product. This is followed by factorizing and resumming outside sets $X$. Since this is a familiar process from [6], we merely write down the result.

$$
\begin{aligned}
& \int_{A} d \mu e^{-\int_{0}^{\beta} V(\tau) d \tau} a_{1}\left(t_{1}\right) \ldots a_{s}\left(t_{s}\right) / \int_{A} d \mu e^{-\int_{0}^{\beta} V(\tau) d \tau} \\
& =\sum_{X, \Gamma} K(X, \Gamma) \int_{(A-X)^{\wedge}} d \mu e^{-\int_{0}^{\beta} V(t) d \tau} / \int_{A} d \mu e^{-\int_{0}^{\beta} V(\tau) d \tau}
\end{aligned}
$$

where

$$
\begin{aligned}
K(X, \Gamma) & =\int_{\left(X-\Gamma^{c}\right)^{\wedge}} d \mu H_{\Gamma} e^{-\rho j V(t) d \tau} \prod_{i} a_{i}\left(t_{i}\right) \\
H_{\Gamma} & =\prod_{\alpha \in \Gamma} H_{\alpha} .
\end{aligned}
$$

If $S \subset \Lambda, \hat{S}=\left\{x \in S\right.$ : dist $\left.(x, \partial S)>\frac{1}{10}\right\} . \partial S=(\bar{S}-\operatorname{Int} S)-\partial \Lambda$. The distinguished sets have been required to include the supports of all the $f_{i j} . \Gamma$ is a subset of $\left\{S_{\alpha}\right\}$ and also denotes the corresponding set in $\mathbb{R}^{3}$. The sum over $X, \Gamma$ in (2.10) runs over 
all $X \subset A$ such that $\bigcup_{j \in J} \Delta_{j} \subset X$ and, for a given $X$, all $\Gamma$ such that (1) $\overline{(\overline{\ln t} \overline{\operatorname{In}})}=\Gamma$

(2) each component of $X-\Gamma^{c}$ contains at least one $\Delta_{j} j \in J . \Gamma^{c}$ is the set of faces $S_{\alpha}$ in $X$, complementary to $\Gamma$, considered as a subset of $\mathbb{R}^{3}$. (The $S_{\alpha}$ are closed sets.) Our constants $c_{\alpha}$ all satisfy

$0<c_{\alpha}<\infty$

and the same notation may mean different constants in different sections if not obviously related.

\section{Results}

Proposition 3.1. Let $Z(A)=\operatorname{Tr}_{A}\left(\exp -\beta H^{A}\right)$ and let $\Lambda_{1} \subset A$ be open. Then, if $|Z(\Lambda)|<\infty$,

$$
\left|Z\left(\Lambda_{1}\right) / Z(\Lambda)\right| \leqq 1 .
$$

Proof. Apply the minimax principle.

Theorem 3.2. If for some $B \geqq 0$

$$
1 / 2 H_{00}+2 V+B N \geqq 0
$$

then for some $\mu_{0}$ (large negative), the cluster expansion (2.10), (2.11) converges uniformly in $\Lambda$ for $\mu_{1}, \ldots, \mu_{l} \leqq \mu_{0}$.

Theorem 3.3. Let $A, B$ be quantities of the type (2.6) and let $B_{\xi}$ for $\xi \in \mathbb{R}^{3}$ denote the translation in the obvious sense of $B$ by $\xi$. For some $\mu_{0}$ (large negative), if (3.1) holds and $\mu_{i} \leqq \mu_{0}$ for $i=1, \ldots, l$, then

$$
\left|\left\langle A B_{\xi}\right\rangle_{A}-\langle A\rangle_{A}\langle B\rangle_{A}\right| \leqq C_{A, B} \exp \left(-c_{\mu_{1}, \ldots, \mu_{l}} \mid \xi\right)
$$

uniformly in $\Lambda$, for $|\xi|$ large. $c_{\mu_{1}, \ldots, \mu_{l}} \rightarrow \infty$ as $\mu_{1}, \ldots, \mu_{l} \rightarrow-\infty$.

Theorem 3.4. There exists $\mu_{0}$ (large negative) such that if $\mu_{i} \leqq \mu_{0}$ for $i=1, \ldots, l$ and (3.1) holds then

(1) $\lim Z\left((\Lambda-X)^{\wedge}\right) / Z(A)$ exists for all $X$.

(2) $\lim _{|A| \rightarrow \infty}^{|A| \rightarrow \infty}\langle A\rangle_{A}$ exists and the limit of (3.2) holds.

Thus the correlation functions exist and cluster exponentially. $|\Lambda|$ represents the volume of $A$. The $A$ 's are boxes (rectangular parallepipeds) centered at the origin whose minimum width goes to infinity, this is understood in the limits in (1) and (2).

Part (2) of Theorem 3.4 follows from part (1), Proposition 3.1, Theorem 3.2 and Theorem 3.3. Part (1) is not difficult, and is proven in Appendix E. The constants $\mu_{0}$ in Theorem 3.2, Theorem 3.3, and Theorem 3.4 are taken to be the same, this can be done at the expense of not using the largest possible value in each theorem.

Parallel to Proposition 5.3, p. 218, in [6] we have 
Proposition 3.5. Under the hypotheses of Theorem 3.2

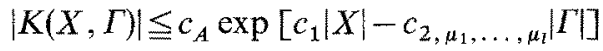

$$
\begin{aligned}
& c_{2, \mu_{1}, \ldots, \mu_{l} \rightarrow \infty} \text { as } \mu_{i} \rightarrow-\infty \text { for } i=1, \ldots, l, c_{1} \text { fixed. }
\end{aligned}
$$

Proposition 3.5 combined with Proposition 3.1 leads to proofs of Theorems 3.2 and 3.3 by the same argument as in [6], which we do not repeat here.

Our proof of Proposition 3.5 uses an inequality of the following type:

Proposition 3.6. For $p>1,1 / p+1 / q=1$

$$
\left|\operatorname{Tr}_{A}\left(\operatorname{Te}^{-\int_{0}^{\beta} H^{\left.A^{(} \tau\right) d \tau}} A\right)\right| \leqq\left[\operatorname{Tr}_{A} e^{-\beta\left(H^{A}+p^{V}\right)}\right]^{1 / p} \cdot\left(\int d|\mu| A^{q}\right)^{1 / q}
$$

This inequality is closely analogous to Holder's inequality in Euclidean Field Theory. It has the important feature that the fermion statistics, or equivalently, the signed measure in (2.8) has been preserved for the first factor on the right. For the absolute value of the measure $\mu$ appearing in the other factor on the right we have:

Proposition 3.7. The absolute value $|\mu|$ of $\mu$ is equal to the measure obtained by changing all fermion species to bosons.

Finally, as an example of a potential $V$ which exploits most of the latitude (see (a) and (b) below Theorem 3.8) of Theorems 3.2, 3.3 and 3.4, set

$$
V=\frac{g^{2}}{2} \int d^{3} x d^{3} y:\left(\bar{\phi}_{1} \phi_{1}-\bar{\phi}_{2} \phi_{2}\right)(x) v(x-y)\left(\bar{\phi}_{1} \phi_{1}-\bar{\phi}_{2} \phi_{2}\right)(y): \text { where } \phi_{1} \equiv \phi \text { is a }
$$

boson field and $\phi_{2} \equiv \varphi$ is a fermion field. $v(x-y)$ is the truncated Coulomb potential

$$
v(x-y)=\int d^{3} z f(x-z) \frac{1}{|x-y|} f(y-z)
$$

where $f$ is a non-negative real $C^{2}$ function on $\mathbb{R}^{3}$ such that $f(x)=0$ for $|x| \geqq 1 / 10$. This $V$ satisfies

Theorem 3.8. For $B \geqq 0$ sufficiently large

$$
1 / 2 H_{00}+2 V+B N \geqq 0 \text {. }
$$

This theorem is the equivalent of the Dyson-Lenard theorem for the Coulomb potential $[2,3,8]$ and shares the following features with it (a) $V$ is not stable in the sense of Ruelle (b) at least one species must obey fermion statistics ((b) is not supposed to be obvious).

\section{Proof of Proposition 3.5}

We use the three lines lemma, thus set

$$
K(X, \Gamma, z)=\int_{X_{1}} d \mu H_{\Gamma} e^{-2 z \int_{0}^{\beta} \mathcal{V}(t) d \tau} \prod_{i} a_{i}^{2-2 z}\left(t_{i}\right)
$$


where $X_{1}=\left(X-\Gamma^{c}\right)^{-}$. To make this analytic in $z$ we temporarily assume that $\int_{X_{1}}$ only integrates over the subset of path space representing less that $\eta_{j}, j=1,2, \ldots, l$ particles of species $j$, and furthermore that $V$ is bounded above and below on this subset. We obtain bounds uniform with respect to these assumptions which can be removed by taking limits at the end of the proof. By the three lines lemma,

$$
|K(X, \Gamma)| \leqq\left(\sup _{\operatorname{Re} z=0}|K(X, \Gamma, z)|\right)^{1 / 2}\left(\sup _{\operatorname{Re} z=1}|K(X, \Gamma, z)|\right)^{1 / 2} .
$$

By taking absolute values inside the $\int d \mu$ integral and then using the Cauchy Schwartz estimate:

$\left(\sup _{\operatorname{Re} z=0}|K(X, \Gamma, z)|\right)^{1 / 2} \leqq\left(\int_{X_{1}} d|\mu| H_{\Gamma}\right)^{1 / 4}\left(\int_{X_{1}} d|\mu| \prod_{i} a_{i}^{4}\left(t_{i}\right)\right)^{1 / 4}$.

Therefore, to prove Proposition 3.5 we derive the following three estimates.

$$
\begin{aligned}
& \int_{X_{1}} d|\mu| \prod_{i} a_{i}^{4}\left(t_{i}\right) \leqq c_{A}^{\prime \prime} e^{c_{3}|X|} \\
& \int_{X_{1}} d|\mu| H_{\Gamma} \leqq c_{4} e^{-4 c_{2, \mu_{1}}, \ldots, \mu_{3}|\Gamma|} e^{c^{\prime \prime}|X|} \\
& \sup _{\operatorname{Re} z=1}|K(X, \Gamma, z)| \leqq c_{5} e^{c_{6}|X|}
\end{aligned}
$$

To prove (4.4), combine Proposition 3.7 with the easy estimate $a_{i} \leqq l N$. The proof of (4.5) is deferred to Appendix D.

Proof of (4.6). Unravel $H_{\Gamma}$ by expanding $H_{\Gamma}=\prod_{\alpha \in \Gamma}\left(1-E_{\alpha}\right)$

$$
\sup _{\operatorname{Re} z=1}|K(X, \Gamma, z)| \leqq \sum_{\Gamma_{1} \subset \Gamma} \sup _{\operatorname{Re} z=1}\left|\int_{X\left(\Gamma_{1}\right)} d \mu e^{-2 z \int_{0}^{\beta} V(z) d t} \prod_{i} a_{i}^{2-2 z}\left(t_{i}\right)\right|
$$

where $X\left(\Gamma_{1}\right)=\left(X-\left(\Gamma^{c} \cup \Gamma_{1}\right)\right)^{\wedge}$

$$
\leqq 2^{|\Gamma|} \sup _{\Gamma_{1} \subset \Gamma \operatorname{Re} z=1} \sup _{X\left(\Gamma_{1}\right)} d \mu e^{-2 z \sum_{0}^{\beta} V(\tau) d \tau} \prod_{i} a_{i}^{2-2 z}\left(t_{i}\right) \mid .
$$

Thus the proof of (4.6) will be completed by

$$
\sup _{\operatorname{Re} z=1}\left|\int_{Y} d \mu e^{-2 z \int_{0}^{\beta} V(\mathrm{z}) d t} \prod_{i} a_{i}^{2-2 z}\left(t_{i}\right)\right| \leqq e^{c_{\gamma}|X|}
$$

uniformly in $Y \subset X$ open. The left hand side of (4.9) may be rewritten as

$$
\sup _{\operatorname{Re} z=1}\left|\operatorname{Tr}_{Y}\left(\operatorname{Te}^{-\int^{-\delta}\left[H_{0}^{Y}+2 z V\right] d \tau} \prod_{i} a_{i}^{2-2 z}\left(t_{i}\right)\right)\right| \text {. }
$$

We refer to the proof of Proposition 3.6 in Appendix C to show that (4.10) is less than

$$
\operatorname{Tr}_{Y}\left(e^{-\beta\left(H_{0}^{X}+2 V\right)}\right)
$$


This is in fact the main point in the proof of Proposition 3.6. It exploits properties of the trace and exponential. By the minimax theorem or equivalently Proposition $3.1,(4.11)$ is less than

$$
\operatorname{Tr}_{X}\left(e^{-\beta\left(H_{0}^{\hat{x}}+2 W\right)}\right)
$$

and this may be estimated by $e^{c_{7}|x|}$ by splitting $H_{0}+2 \mathrm{~V}$ into $1 / 2 H_{0}, 1 / 2 H_{0}+2 \mathrm{~V}$ and using the well known fact,

$$
\operatorname{Tr}\left(e^{-A}\right) \leqq \operatorname{Tr}\left(e^{-B}\right)
$$

if $A \geqq B$, along with the hypothesis (3.1).

\section{Appendix A. $\mid$ Fermion Measure $\mid=$ Boson Measure}

Let $\left(x_{1}, \ldots, x_{N}\right)$ and $\left(y_{1}, \ldots, y_{N}\right)$ be two sets of distinct points in $R^{3}$. We denote a single particle path space measure for paths from $x$ to $y$ in time $a \leqq t \leqq \mathrm{~b}$ by

$$
\int d \mu_{x, y}^{a, b}
$$

The path is described by $z(t)$. We construct the boson and fermion measures as follows:

$$
\mu_{F, B}=(1 / N !) \sum_{P} \varepsilon^{S(P)} \prod_{i}\left(\int d \mu_{y P_{(i)}, x_{i}}^{a, b}\right)
$$

where $S(P)$ is $\left(\begin{array}{l}0 \\ 1\end{array}\right)$ if the permutation is $\left(\begin{array}{c}\text { even } \\ \text { odd }\end{array}\right)$ and $\varepsilon=1$ for bosons, giving $\mu_{B}$, and $\varepsilon=-1$ for fermions, giving $\mu_{F}$. Let $\Xi$ be the space whose points are sets of $N$ points in $R^{3} ; T$ be the space of mappings of $[a, b]$ into $\Xi$. The set $z_{1}(t), \ldots, z_{N}(t) \in \Xi$ identifies the $n$-paths in (A.1) with points in $T$, and $\mu_{F}$ and $\mu_{B}$ are defined (at last) as measures on $T$. The image in $T$ of continuous paths that never intersect each other we call $T^{\prime} . T-T^{\prime}$ is a set of measure zero. The sum in (A.1) realizes $\mu_{F}$ and $\mu_{B}$ as a sum of measures with disjoint supports in $T^{\prime}$. Thus $\left|\mu_{F}\right|=\mu_{B}$.

\section{Appendix B. The Truncated Coulomb Interaction and Its Stability}

We consider

$$
H=H_{0 F}+H_{O B}+g^{2} / 2 \int:(\bar{\psi} \psi-\bar{\phi} \phi) v(\bar{\psi} \psi-\bar{\phi} \phi):
$$

with

$$
\begin{array}{rlrl}
H_{O F} & =\int\left(-\frac{1}{2 m} \bar{\psi} \Delta \psi\right), & N_{F}=\int \bar{\psi} \psi \\
H_{0 B} & =\int\left(-\frac{1}{2 M} \bar{\phi} \Delta \phi\right), & N_{B}=\int \bar{\phi} \phi \\
N & =N_{F}+N_{B}
\end{array}
$$

$v$ is our truncated $1 / r$ potential given by

$$
v(x-y)=\int d^{3} z f(x-z)|x-y|^{-1} f(y-z)
$$


We assume $f$ is a non-negative real $C^{2}$ function on $R^{3}$ satisfying

$$
f(x)=0 \quad \text { if }|x|>1 / 10
$$

We also define auxiliary potentials $v_{n}$

$$
v_{n}(x-y)=\int d^{3} z f(x-z)|x-y|^{-1} e^{-n|x-y|} f(y-z)
$$

$v$ and $v_{n}$ satisfy the following properties:

$$
\begin{array}{ll}
\text { P.0 } & v=v_{0} \\
\text { P.1 } & v_{n}(x)=0 \text { if }|x| \geqq 2 / 10 \\
& \left|v_{n}(x)\right| \leqq c_{1}|x|^{-1} e^{-n|x|}
\end{array}
$$

P.2 There are $c_{2}>0$ and $c_{3}>0$ such that

$$
v_{n}(x) \geqq c_{2}|x|^{-1} e^{-n|x|} \quad \text { if } \quad c_{3} \geqq|x|
$$

and $v_{m}(x) \geqq 0$ for all $x$.

P.3 There is $c_{4}$ such that

$$
v_{n} * v_{n}(x) \leqq c_{4}\left(r^{-1} e^{-n r}\right) *\left(r^{-1} e^{-n r}\right)
$$

P.4 If $n>m$ then

$$
v_{m}-v_{n} \geqq 0
$$

as an operator and numerically.

P.5 There is a $c_{5}$ such that

$$
\left(v-v_{n}\right)(0)=c_{5} n
$$

P.6 Let $\left\{\chi_{i}\right\}$ be translates over a lattice of a real function in $C_{0}^{2}$, then there is a $c_{6}>0\left(c_{6}\right.$ depending on $\left.\chi_{i}\right)$ such that

$$
v-v_{1} \geqq c_{6} \sum \chi_{i} \chi_{i}
$$

as an operator inequality.

These properties are immediate except for P.6. It is proved below.

Theorem. If to $v(x)$ may be associated a set of potentials $v_{n}(x)$ satisfying $\mathbf{P} .0$ through P.6 then $\mathrm{H}$ as given in (B.1) is stable.

A proof of this theorem may be constructed by examining the proof in [3] and verifying these properties are sufficient to provide stability. In the absence of the exclusion principle - that is if $\psi$ were a boson field instead of a fermion field - the Hamiltonian is unstable. By examining [1] one can deduce if

$$
H+B N^{\gamma} \geqq 0
$$

then $\gamma \geqq 7 / 5$.

Proof of P.6. We wish to prove P.6, that for $\chi_{i}$ translates over a lattice of a real function in $C_{0}^{2}$

$$
\int f\left(r^{-1}\left(1-e^{-r}\right)\right) f \geqq c \sum \chi_{i} \chi_{i}
$$


Basically we proceed via a few reductions. Assume $\left\{\phi_{i \alpha}\right\}$ are real functions, $\{\alpha\}$ a finite indexing set, and for fixed $\alpha$ the $\phi_{i \alpha}$ are translates over a lattice of each other; then if

$$
\chi_{i}=\sum_{\alpha} \phi_{i \alpha}
$$

it follows that (B.14) is implied by

$$
\int f\left(r^{-1}\left(1-e^{-r}\right)\right) f \geqq c^{\prime} \sum_{i, \alpha} \phi_{i \alpha} \phi_{i \alpha}
$$

This is the first reduction. It follows from the inequality

$$
\phi_{i \alpha} \phi_{i \beta}+\phi_{i \beta} \phi_{i \alpha} \leqq \phi_{i \alpha} \phi_{i \alpha}+\phi_{i \beta} \phi_{i \beta}
$$

upon expanding

$$
\sum \chi_{i} \chi_{i}=\sum\left(\sum_{\alpha} \phi_{i \alpha}\right)\left(\sum_{\beta} \phi_{i \beta}\right)
$$

The next reduction is to observe that (B.16) follows from the relationship

$$
f(z-x) \frac{1-e^{-|x-y|}}{|x-y|} f(z-y) \geqq c^{\prime \prime} \phi_{i \alpha} \phi_{i \alpha}
$$

for $z \in U_{\alpha}$, the $U_{\alpha}$ non-empty open sets; $i$ fixed. This can be seen by noting that the integral in (B.16) then contains positive contributions to dominate the terms on the right hand side (which may be picked coming from disjoint portions of the integral).

We look at an equivalent form of (B.18) again for $z \in U_{\alpha}$,

$$
\frac{1-e^{-|x-y|}}{|x-y|} \geqq c^{\prime \prime} \frac{1}{f(z-x)} \phi_{i \alpha}(x) \phi_{i \alpha}(y) \frac{1}{f(z-y)}
$$

From the proof of Fact 5 in [3] we get (B.19) provided $\frac{1}{f(z-x)} \phi_{i \alpha}(x)$ is in $C_{0}^{2}$, with derivative estimates uniform in $z$, for $z \in U_{\alpha}$, The $\phi_{i \alpha}$ are easily constructed as a finite $C_{0}^{2}$ partition of $\chi_{i}$ satisfying

$$
\operatorname{Supp}\left(\phi_{i \alpha}(x)\right) \subset\{x \mid f(x-z)>\varepsilon\}
$$

for some $\varepsilon>0$ and $z$.

\section{Appendix C. Proof of Proposition 3.6}

As in Section 4, the three lines lemma implies (the comments below (4.1) are in force)

$$
\begin{aligned}
& \left|\operatorname{Tr}_{A}\left(\operatorname{Te}^{-\int_{0}^{-\beta} H^{A}(\tau) d \tau} A\right)\right| \\
& \leqq\left(\sup _{\operatorname{Re} z=1}\left|\operatorname{Tr}_{A}\left(\operatorname{Te}^{\left.-\int_{0}^{-\left[H_{0}+z p V\right](\tau) d \tau} A^{(1-z) q}\right)}\right)\right|\right)^{1 / p}\left(\int_{A} d|\mu| A^{q}\right)^{1 / q}
\end{aligned}
$$


where $1 / p+1 / q=1, p>1$, [9]. To complete the proof we need to show that the first factor on the right of (C.1) is less than

$$
\left(\operatorname{Tr}_{A}\left(e^{-\int_{0}^{\beta}\left[H_{0}+p V\right](\tau) d \tau}\right)\right)^{1 / p}
$$

The principle involved is contained in the following lemma.

Lemma. Let $A, B$ be hermitian matrices with $A \geqq 0$. Let $s_{1}, \ldots, s_{n} \geqq 0$ with $\sum_{i=1}^{n} s_{i}=1$ and let $u_{1}, \ldots, u_{n-1}$ be unitary matrices, then

$$
\begin{aligned}
& \left|\operatorname{Tr}\left(e^{-s_{1}(A+i B)} u_{1} e^{-s_{2}(A+i B)} u_{2} \ldots u_{n-1} e^{-s_{n}(A+i B)}\right)\right| \\
& \leqq \operatorname{Tr} e^{-A} .
\end{aligned}
$$

Proof. It is sufficient to prove it when $s_{1}, \ldots, s_{n}$ are rational fractions with $N_{0}$ their common denominator. Apply the Trotter product formula in the form

$$
e^{-s_{k}(A+i B)}=\lim _{l \rightarrow \infty}\left(e^{-\left(1 / l N_{0}\right) i B} e^{-\left(1 / l N_{0}\right) A}\right)^{l N_{0} s_{k}}
$$

for $k=1,2, \ldots, n$ so that the left hand side of (C.3) is

$$
\lim _{l \rightarrow \infty} \operatorname{Tr}\left(\prod_{j=1}^{l N_{0}}\left(V_{j} e^{-\left(1 / l N_{0}\right) A}\right)\right)
$$

Where $V_{j}$ is a unitary operator (either $e^{-\left(1 / l N_{0}\right) i B}$ or $u_{k} e^{-\left(1 / l N_{0}\right) i B}$ for some $\left.k\right)$. By Holder's inequality for trace norms [9], the absolute value of (C.4) is less than

$$
\begin{aligned}
& \lim _{l \rightarrow \infty} \prod_{j=1}^{l N_{0}}\left(\operatorname{Tr}\left|V_{j} e^{-\left(1 / l N_{0}\right) A}\right|^{l N_{0}}\right)^{1 / l N_{0}} \\
& =\lim _{l \rightarrow \infty} \prod_{j=1}^{l N_{0}}\left(\operatorname{Tr}\left|e^{-\left(1 / l N_{0}\right) A}\right|^{l N_{0}}\right)^{1 / l N_{0}} \\
& =\operatorname{Tr}\left(e^{-A}\right)
\end{aligned}
$$

which concludes the proof of the lemma. We do not discuss the technicalities involved in extending the inequality (C.3) to allow $A=H_{0}+p V, B=p(\operatorname{Im} z) V$, and $u_{i}=a_{i}^{i[\operatorname{Im}(1-z)] q}$, thereby obtaining (C.2).

\section{Appendix D. A Path Space Estimate Incorporating Conditions that Paths Must Hit Barriers}

We study

$$
I=\int_{\hat{\tilde{x}}} d \mu H_{\Gamma}
$$

We restrict our notation to the situation where a single boson species is described by the measure, this is a trivial simplification. Without the function $H_{r}$ this would be the integral over $n$-paths in $\hat{X}$ that realizes the trace of $e^{-\rho r} \hat{x}$. The inclusion of $H_{\Gamma}$ restricts the integral to $n$-paths with the property that each barrier 
in $\Gamma$ is hit by some path (different barriers may be hit by different paths or the same path).

We majorize (D.1) by a sum of terms, one for each partition $p$ of the faces in $\Gamma$

$$
p \leftrightarrow\left(p_{1}, \ldots, p_{\mathrm{s}}\right)
$$

$p_{i}$ a subset of faces in $\Gamma$. To each $p_{i}$ is associated a path integral from $x_{i}$ to $y_{i}$, the $x_{i}$ and $y_{i}$ localized in $\Delta_{j_{i}}$ and $\Delta_{j_{i}}$. The paths associated to $p_{i}$ must hit all the barriers in $p_{i}$. With this notation we claim

$$
\begin{gathered}
I \leqq \sum_{p} \sum_{j_{i}, j_{i}{ }_{i}} \prod_{i=1}^{s}\left(\int_{\Delta_{j_{i}}} d x_{i} \int_{\Delta_{S_{i}^{\prime}}} d y_{i} \int_{p_{i}} d \mu_{x_{i}, y_{i}}^{\beta}\right) \\
\cdot \operatorname{Tr}_{\hat{X}}\left(\prod \bar{\phi}\left(y_{i}\right) e^{-\beta H_{\hat{\gamma}}^{\hat{X}}} \prod \phi\left(x_{i}\right)\right)
\end{gathered}
$$

Realized as $n$-paths the trace in (D.3) is greater than $I$ since all the $n$-paths in $I$ are summed over with same numerical weight, but some more than once.

We now note that the expression inside the trace equals

$$
\prod \bar{\phi}\left(y_{i}\right) e^{-N} e^{-\beta H_{0}^{\hat{x}}+2 N} e^{-N} \prod \phi\left(x_{i}\right)
$$

We let $\alpha_{j}$ be the number of $x_{i}$ localized in $A_{j}$, and $\beta_{j}$ the number of $y_{i}$ localized in $\Delta_{j}$. We recall if $T \geqq 0$ then

$$
\left|\operatorname{Tr}\left(e^{-T} R\right)\right| \leqq \operatorname{Tr}\left(e^{-T}\right) \cdot\|R\|
$$

Our " $R$ " is of the form

$$
\prod\left(\int_{\Delta_{j_{i}}} d x_{i} \int_{\Delta_{j_{i}^{\prime}}} d y_{i}\right) G\left(x_{i}, y_{i}\right) e^{-N} \prod \phi\left(x_{i}\right) \prod \bar{\phi}\left(y_{i}\right) e^{-N}
$$

Normal ordering and employing $N_{\tau}$ estimates one finds

$$
\|\left(\text { D.6) } \| \leqq \prod\left(\alpha_{j}+1\right)^{2\left(\alpha_{j}+1\right)}\left(\beta_{j}+1\right)^{2\left(\beta_{j}+1\right)} \cdot \operatorname{Sup}\left|G\left(x_{i}, y_{i}\right)\right|\right.
$$

We have used the fact that the integration regions are of volume one, so that the sup norm dominates the $L_{2}$ norm (and other norms arising in the process).

We let $h_{i}=h\left(p_{i}, \Delta_{j_{i}}, \Delta_{j_{i}}\right)$ be the maximum over $x_{i}$ and $y_{i}$ of the path integrals in parentheses in (D.3). This yields the estimate

$$
I \leqq e^{c_{1}|X|} \sum_{p} \sum_{j_{i}, j_{i}} \prod\left(\alpha_{j}+1\right)^{2\left(\alpha_{j}+1\right)}\left(\beta_{j}+1\right)^{2\left(\beta_{j}+1\right)} \prod h_{i}
$$

We write

$$
h_{i} \leqq h_{1 i} \cdot h_{2 i} \cdot h_{3 i}^{2}
$$

with

$$
h_{1 i}=c_{3} e^{-c_{4} d\left(\Delta_{j_{i}}, S_{\alpha}\right)}, S_{\alpha} \in p_{i}
$$

and

$$
h_{2 i}=c_{3} e^{-c_{4} d\left(\Delta_{j_{i}} \cdot S_{\left.\alpha^{\prime}\right)}\right)}, S_{\alpha^{\prime}} \in p_{i}
$$

where $S_{\alpha}$ and $S_{\alpha^{\prime}}$ are picked minimizing the distance $d$. We get that

$$
\operatorname{Sup}_{p} \sum_{j_{i,}, j_{i}^{\prime}}\left(\alpha_{j}+1\right)^{2\left(\alpha_{j}+1\right)}\left(\beta_{j}+1\right)^{2\left(\beta_{j}+1\right)} \prod h_{1 i} \prod h_{2 i} \leqq e^{c 5|\Gamma|}
$$


by an argument as in Section 10 of [6]. Thus we have the estimate

$$
I \leqq e^{c_{1}|X|+c_{5}|\Gamma|} \sum_{p} \prod h_{3 i}^{2}
$$

The final result is obtained provided

$$
\operatorname{Sup}_{p} \prod h_{3 i} \leqq e^{-c_{6}|\Gamma|}
$$

with $c_{6}$ going to infinity with $\mu$ going to minus infinity, and

$$
\sum_{p} \prod h_{3 i} \leqq e^{c_{6}^{\prime}|\Gamma|}
$$

The estimate for $h_{3 i}^{2}$, the heart of the matter, is obtained by the same argument as in Proposition 8.1 of [6]. It is the square root of the measure for paths hitting all the barriers in $p_{i}$ in time $\beta$. (The root is taken so that $h_{1 i}$ and $h_{2 i}$ may be factored out of the total probability.) The length of such a path must be at least $c_{7}\left|p_{i}\right|$ for $\left|p_{i}\right|$ large. It is not surprising that one gets

$$
h_{3 i} \leqq e^{-1 / 4 \mu \beta+c_{9}\left|p_{i}\right|-\varepsilon_{8}\left|p_{i}\right|^{2}}\left|p_{i}\right| \text { ! }
$$

The factorial accomodates different orders of hitting the barriers. By picking $\mu$ large enough one gets (D.13) for any $c_{6}$.

To get (D.13)' we observe that to a path that hits barrier $i$ and then barrier $j$ may be associated a numerical factor $e^{-\varepsilon^{\prime} d_{i j}}$, where $d_{i j}$ is the distance between barrier $i$ and barrier $j$, such that $\sum_{p} \prod h_{3 i}$ is overestimated by

$$
\prod_{\alpha \in I} c^{\prime \prime}\left(1+\sum_{\beta} e^{-c^{\prime} d_{\alpha \beta}}\right) \leqq e^{c^{\prime}|\Gamma|}
$$

(Those paths contributing to $h_{3 i}$ required to hit barriers $1,2, \ldots, k$ in order, have associated to them

$$
\left.\left(c^{\prime \prime}\right)^{k} \prod_{j=1}^{k-1} e^{-c^{\prime} d_{j j+1}}\right)
$$

\section{Theorem.}

$$
I \leqq e^{c_{1}|X|-k|\Gamma|}
$$

where $k$ can be made arbitrarily large, $c_{1}$ fixed, by picking $\mu$ large negative.

\section{Appendix E. Proof of Theorem 3.4 (1)}

We consider the difference between the ratio of $Z$ s in Theorem 3.4 (1) for two choices of $A, \Lambda_{1}$, and $\Lambda_{2}$.

$$
\begin{aligned}
& Z\left(\left(\Lambda_{1}-X\right)^{\wedge}\right) / Z\left(\Lambda_{1}\right)-Z\left(\left(\Lambda_{2}-X\right)^{\wedge}\right) / Z\left(\Lambda_{2}\right)= \\
& =\left(Z\left(\Lambda_{2}\right) Z\left(\left(\Lambda_{1}-X\right)^{\wedge}\right)-Z\left(\Lambda_{1}\right) Z\left(\left(\Lambda_{2}-X\right)^{\wedge}\right)\right) / Z\left(\Lambda_{1}\right) Z\left(\Lambda_{2}\right) .
\end{aligned}
$$

We pick a set $\left\{\Delta_{j}, j \in J\right\}$ of distinguished cubes with the property that their union is inside $(\Lambda-X)^{\wedge}$ for all $A$ large enough, and such that this union separates $X$ 
and the component of infinity. This choice depends on $X$ but is independent of $A_{1}$ and $A_{2}$ for $A_{1}$ and $A_{2}$ large enough. ( $U_{j \in J} A_{j}$ is a collar around $X$.)

We view each product of $Z$ 's in the numerator of the right term in (E.1) as a single partition function for a doubled system, each subsystem with the same interactions as the original system, but with no mutual interactions. The boundary data of the two subsystems are different to yield the indicated products. We expand each product of $Z$ s in the (E.1) numerator in a single cluster expansion for the doubled systems, using the distinguished cubes defined above.

Pairs $(X, \Gamma)$ arising in the two cluster expansions cancel until $X$ hits either $\partial \Lambda_{1}$ or $\partial \Lambda_{2}$. Thus the difference in (E.1) goes to zero exponentially with the minimum width of $A_{1}$ or $A_{2}$ whichever is smaller, provided $\mu_{0}$ is large enough negative.

This proof is similar to the proof of clustering in [6], Section 4, which also uses a doubled system.

\section{References}

1. Dyson, F. J.: J, Math. Phys. 8, 1538 (1967)

2. Dyson,F.J., Lenard, A.: J. Math. Phys. 8, 423 (1967); J. Math. Phys. 9, 698 (1968)

3. Federbush, P.: J. Math. Phys, 16, 347 (1975); J. Math. Phys. 16, 706 (1975)

4. Federbush, P.: J. Math. Phys. 17, 200 (1976); J. Math. Phys, 17, 204 (1976)

5. Ginibre, J: Some Applications of Functional Integration in Statistical Mechanics. In: Statistical Mechanics and Quantum Field Theory, Les Houches 1970 (ed. C. Dewitt, R. Stora). New York: Gordon and Breach, 1971

6. Glimm, J., Jaffe, A., Spencer, T.: The Cluster Expansion. In: Constructive Quantum Field Theory, The 1973 "Ettore Majorana" International School of Mathematical Physics, (ed. G. Velo, A. Wightman). Berlin-Heidelberg-New York: Springer 1973

7. Lieb, E.H., Lebowitz, J.L.: Advan. Math. 9, 316 (1972)

8. Lieb, E.H., Thirring, W. E.: Phys. Rev. Letters 35, 687 (1975)

9. Reed,M., Simon, B.: Fourier Analysis and Self-Adjointness, New York: Academic Press, 1975

Communicated by A. S. Wightman

Received November 11, 1975; in revised form April 6, 1976 